\title{
Expression of Glutamine Metabolism-Related and Amino Acid Transporter Proteins in Adrenal Cortical Neoplasms and Pheochromocytomas
}

\author{
Hye Min Kim and Ja Seung Koo \\ Department of Pathology, Yonsei University College of Medicine, Seoul, Republic of Korea \\ Correspondence should be addressed to Ja Seung Koo; kjs1976@yuhs.ac
}

Received 2 April 2020; Revised 17 December 2020; Accepted 23 December 2020; Published 6 January 2021

Academic Editor: Carlo Chiarla

Copyright (c) 2021 Hye Min Kim and Ja Seung Koo. This is an open access article distributed under the Creative Commons Attribution License, which permits unrestricted use, distribution, and reproduction in any medium, provided the original work is properly cited.

\begin{abstract}
Background. Glutamine metabolism is considered an important metabolic phenotype of proliferating tumor cells. Objective. The objective of this study was to investigate the expression of glutamine metabolism-related and amino acid transporter proteins in adrenal cortical neoplasms (ACNs) and pheochromocytomas (PCCs) in the adrenal gland. Methods. A tissue microarray was constructed for 132 cases of ACN (115 cases of adrenal cortical adenoma and 17 cases of adrenal cortical carcinoma) and 189 cases of PCC. Immunohistochemical staining for glutamine metabolism-related proteins GLS1 and GDH and amino acid transporter proteins SLC1A5, SLC7A5, and SLC7A11 as well as SDHB was performed and compared with clinicopathologic parameters. Results. The expression levels of GLS $(p<0.001)$, SLC7A5 $(p=0.049)$, and SDHB $(p=0.007)$ were higher in ACN than in PCC, whereas the expression levels of SLC1A5 $(p<0.001)$ and SLC7A11 $(p<0.001)$ were higher in PCC than in ACN. In ACN, GLS positivity was associated with a higher Fuhrman grade $(p=0.009)$, and SLC1A5 positivity was associated with SDHB positivity $(p=0.004)$ and a clear cell proportion $<25 \%(p=0.010)$. SDHB negativity was also associated with tumor cell necrosis $(p=0.007)$. In PCC, SLC7A11 positivity was associated with nonnorepinephrine type $(p=0.008)$. In Kaplan-Meier analysis, patients with GLS positivity $(p=0.039)$ and SDHB negativity $(p=0.005)$ had significantly shorter overall survival in ACN. In PCC patients with a GAPP score $\geq 3$, GLS positivity $(p=0.001)$ and SDHB positivity $(p=0.001)$ were associated with shorter disease-free survival, whereas GLS positivity $(p=0.004)$ was also associated with shorter overall survival. Conclusions. The expression of glutamine metabolism-related and amino acid transporter proteins in ACN and PCC is distinct and associated with prognosis.
\end{abstract}

\section{Introduction}

Typical tumors in the adrenal gland are adrenal cortical neoplasms (ACNs) arising from the adrenal cortex and pheochromocytomas (PCCs) from the adrenal medulla. ACNs comprise benign adrenal cortical adenomas (ACAs) and malignant adrenal cortical carcinomas (ACCs). ACA is a relatively common tumor, whereas ACC is an extremely rare tumor with an annual incidence of $1-2$ cases per 1 million people with a highly aggressive phenotype $[1,2]$. PCC is a neuroendocrine tumor that occurs in the chromaffin cells of the adrenal medulla, found in 2-8 cases per 1 million people [3]. Although ACNs and PCCs can be differentiated by dis- parities in the originating cells, histologic findings are similar and the behavior of these tumors cannot be adequately predicted based on histologic findings alone.

An important part of the metabolic pathway for tumor maintenance and growth is glutamine metabolism and amino acid transport. In cell culture and implanted tumor studies, cancer cells were found to metabolize glutamine better than other amino acids $[4,5]$. Therefore, glutamine metabolism is considered an important metabolic phenotype of proliferating tumor cells. Glutamine metabolism contributes to two important factors in tumor cell proliferation: the production of adenosine triphosphate (ATP) and supplementation of intermediates for macromolecular synthesis. 
Proteins that play an important role in this glutamine metabolic pathway are amino acid transporter-2 (ASCT2), which is involved in the intracellular transport of glutamine consumed by tumor cells, glutaminase 1 (GLS1), an enzyme that converts glutamine to glutamate, and glutamate dehydrogenase (GDH), which converts glutamate into $\alpha$-ketoglutarate to affect the TCA cycle [6-8].

Amino acids are essential for the growth and survival of tumor cells. Among them, essential amino acids such as Thr, Met, Phe, Trp, Val, Ile, Leu, and Lys must be obtained from outside the body, because they cannot be synthesized internally [9-11]. In contrast, even though nonessential amino acids can be synthesized within cells, when their demand is significantly increased by proliferating tumor cells, additional supply is required from extracellular spaces $[12,13]$. Among various membrane-bound solute carrier (SLC) transporters, SLC1, SLC3, SLC6, SLC7, SLC15, SLC17, SLC18, SLC25, SLC26, SLC32, SLC36, and SLC38 are involved in the transport of amino acids. Although most amino acid transporters are expressed in a tissue- and developmentally specific manner in normal cells, the expression of specific amino acid transporters is generally higher in tumor cells, depending on the tumor type than in normal cells [9, 14-16].

Nonetheless, glutamine metabolism-related and amino acid transporter proteins in ACNs and PCCs in the adrenal gland have been poorly studied. The aim of this study was to investigate the expression of glutamine metabolismrelated and amino acid transporter proteins in ACNs and PCCs and to determine the clinical implications.

\section{Materials and Methods}

2.1. Patient Selection. Formalin-fixed paraffin-embedded surgical tissue samples from patients diagnosed with ACN and PCC were obtained from Severance Hospital from January 2000 to December 2012. All cases were retrospectively reviewed by pathologists (Koo JS and Kim HM), and a histologic evaluation was performed on hematoxylin-eosin(H\&E-) stained slides. ACNs were examined using parameters corresponding to the Weiss criteria [17, 18], and PCCs and its catecholamine types were examined using parameters of the GAPP scoring system [19]. Disease-free survival (DFS) was calculated from the date of the first curative surgery to the date of the first locoregional or systemic relapse, or death without any relapse. Overall survival (OS) was estimated from the date of the first curative operation to the date of the last follow-up or death from any cause. Clinicopathologic parameters evaluated in each tumor included patient age at initial diagnosis, tumor recurrence, distant metastasis, and patient survival. The study was approved by the Institutional Review Board of Severance Hospital.

2.2. Tissue Microarray. Representative areas were selected on $\mathrm{H} \& \mathrm{E}$-stained slides, and a corresponding spot was marked on the surface of the matching paraffin block. Core biopsies, $5 \mathrm{~mm}$ each, were taken from selected areas and placed into a $5 \times 4$ recipient block. More than two tissue cores were extracted from each case to minimize extraction bias. Each tissue core was assigned a unique tissue microarray location number that was linked to a database containing other clinicopathologic data.

2.3. Immunohistochemistry. The information of antibodies used for immunohistochemistry are listed in Supplementary Table 1. Succinate dehydrogenase subunit B (SDHB) and BCL2/adenovirus $\mathrm{E} 1 \mathrm{~B} 19 \mathrm{kDa}$ protein-interacting protein 3 (BNIP3) staining were also performed to assess SDHB mutation and tumor hypoxia. Immunohistochemistry was performed on formalin-fixed, paraffin-embedded tissue sections using an automatic immunohistochemistry staining device (Benchmark XT, Ventana Medical System, Tucson, AZ, USA). Briefly, $5 \mu \mathrm{m}$ thick formalin-fixed paraffinembedded tissue sections were transferred to adhesive slides and dried at $62^{\circ} \mathrm{C}$ for 30 minutes. Standard heat epitope retrieval was performed for 30 minutes in ethylene diamine tetraacetic acid, $\mathrm{pH} 8.0$, in an autotimer. The samples were then incubated with primary antibodies. After incubation with primary antibodies, the sections were incubated with biotinylated anti-mouse immunoglobulins, peroxidaselabeled streptavidin (LSAB kit, DakoCytomation), and 3,30diaminobenzidine. Negative control samples were processed without the primary antibody. A positive control tissue was used per the manufacturer's recommendation. Slides were counterstained with Harris hematoxylin.

2.4. Interpretation of Immunohistochemical Staining. All immunohistochemical markers (GLS1, GDH, SLC1A5, SLC7A5, SLC7A11, SDHB, and BNIP3) were accessed by light microscopy. Expression of the included markers was semiquantitatively evaluated using stained slides, as previously described [20]. Tumor cell staining was assessed using the following criteria: 0 , negative or weak immunostaining in $<1 \%$ of the tumor; 1 , focal expression in $1-10 \%$ of tumors; 2 , positive in $11-50 \%$ of tumors; and 3, positive in $51-100 \%$ of tumors. The entire area of each tumor was evaluated, and a score of $\geq 2$ determined by both pathologists was defined as immunohistochemical staining positivity, whereas the score of $<2$ was considered negativity.

2.5. Statistical Analysis. Data were analyzed using IBM SPSS Statistics for Windows, Version 21.0 (Released 2012; IBM Corp., Armonk, NY, USA). To determine the statistical significance of differences, Student's $t$-test and Fisher's exact test were used for continuous and categorical variables, respectively. Statistical significance was set at $p<0.05$. KaplanMeier survival curves and log-rank statistics were employed to evaluate the clinical significance of clinicopathologic markers and the DFS and OS of the patients.

\section{Results}

3.1. Basal Characteristics of Patients. We included a total of 115 cases of ACA and 17 cases of ACC in this study. The basal characteristics of these patients are presented in Supplementary Table 2. Patient age $(p=0.048)$ and tumor size $(p<0.001)$ were significantly different between the two groups. Parameters comprising the Weiss criteria were also significantly different between the two groups (all $p<0.001$, 
TABLE 1: Expression of glutamine metabolism-related proteins and amino acid transporter proteins in adrenal gland neoplasm.

\begin{tabular}{|c|c|c|c|c|}
\hline Parameters & $\begin{array}{c}\text { Total } \\
N=321(\%)\end{array}$ & $\begin{array}{c}\text { Adrenal cortical neoplasm } \\
n=132(\%)\end{array}$ & $\begin{array}{c}\text { Pheochromocytoma } \\
n=189(\%)\end{array}$ & $p$ value \\
\hline GLS & & & & $<0.001$ \\
\hline Negative & $248(77.3)$ & $85(64.4)$ & $163(86.2)$ & \\
\hline Positive & $73(22.7)$ & $47(35.6)$ & $26(13.8)$ & \\
\hline GDH & & & & 0.956 \\
\hline Negative & $316(98.4)$ & $130(98.5)$ & $186(98.4)$ & \\
\hline Positive & $5(1.6)$ & $2(1.5)$ & $3(1.6)$ & \\
\hline SLC1A5 & & & & 0.049 \\
\hline Negative & $242(75.4)$ & $107(81.1)$ & $135(71.4)$ & \\
\hline Positive & 79 (24.6) & 25 (18.9) & $54(28.6)$ & \\
\hline SLC7A5 & & & & 0.007 \\
\hline Negative & $316(98.4)$ & $127(96.2)$ & $189(100.0)$ & \\
\hline Positive & $5(1.6)$ & $5(3.8)$ & $0(0.0)$ & \\
\hline SLC7A11 & & & & $<0.001$ \\
\hline Negative & $259(80.7)$ & $126(95.5)$ & $133(70.4)$ & \\
\hline Positive & $62(19.3)$ & $6(4.5)$ & $56(29.6)$ & \\
\hline SDHB & & & & $<0.001$ \\
\hline Negative & $189(58.9)$ & $47(35.6)$ & $142(75.1)$ & \\
\hline Positive & $132(41.1)$ & $85(64.4)$ & 47 (24.9) & \\
\hline BNIP3 & & & & $<0.001$ \\
\hline Negative & $293(91.3)$ & $111(84.1)$ & $182(96.3)$ & \\
\hline Positive & $28(8.7)$ & $21(15.9)$ & 7 (3.7) & \\
\hline
\end{tabular}

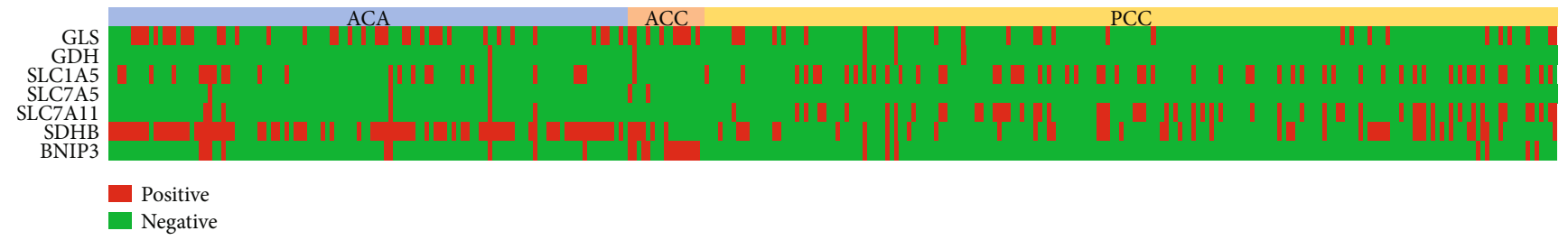

FIGURE 1: Heat map of glutamine metabolism-related and amino acid transporter proteins found in adrenal gland neoplasms (ACA: adrenal cortical adenoma; ACC: adrenal cortical carcinoma; PCC: pheochromocytoma).

Supplementary Table 2). One-hundred and eighty-nine cases of PCC were included, and the basal characteristics of these patients are presented in Supplementary Table 3.

\subsection{Differential Expression of Glutamine Metabolism-Related} and Amino Acid Transporter Proteins, SDHB, and BNIP3 in Adrenal Gland Neoplasms. GLS $(p<0.001)$, SLC7A5 $(p=0.007)$, SDHB $(p<0.001)$, and BNIP3 $(p<0.001)$ were highly expressed in ACNs, whereas SLC1A5 $(p=0.049)$ and SLC7A11 $(p<0.001)$ were highly expressed in PCCs (Table 1). The heat map regarding the expression of immunohistochemical markers and the representative images of the antibody staining are shown in Figures 1 and 2. There was no difference in the expression of glutamine metabolism-related and amino acid transporter proteins between ACAs and ACCs, and the expression of SDHB was lower and BNIP3 was higher in ACCs (Table 2).

We divided our patients with PCC into the SDHBpositive group and SDHB-negative group according to
SDHB positivity. Patients with SDHB positivity revealed to have higher expression of GLS $(p<0.001)$, SLC1A5 $(p=0.014)$, and SLC7A11 $(p=0.001)$ compared to those with SDHB negativity (Table 3 ).

3.3. Correlation between the Expression Levels of Glutamine Metabolism-Related and Amino Acid Transporter Proteins and SDHB with Clinicopathologic Factors. In ACN, GLS positivity was associated with a higher Fuhrman grade $(p=0.009)$, and SLC1A5 positivity was associated with SDHB positivity $(p=0.004)$ and a clear cell proportion of $<$ $25 \%(p=0.010)$. Furthermore, SDHB negativity was associated with tumor cell necrosis $(p=0.007)$. In PCC, SLC7A11 positivity was associated with nonnorepinephrine-type tumors $(p=0.008)$ (Figure 3).

3.4. Expression of Glutamine Metabolism-Related Proteins and Amino Acid Transporter Proteins according to BNIP3. Considering that tumor microenvironment could be related 


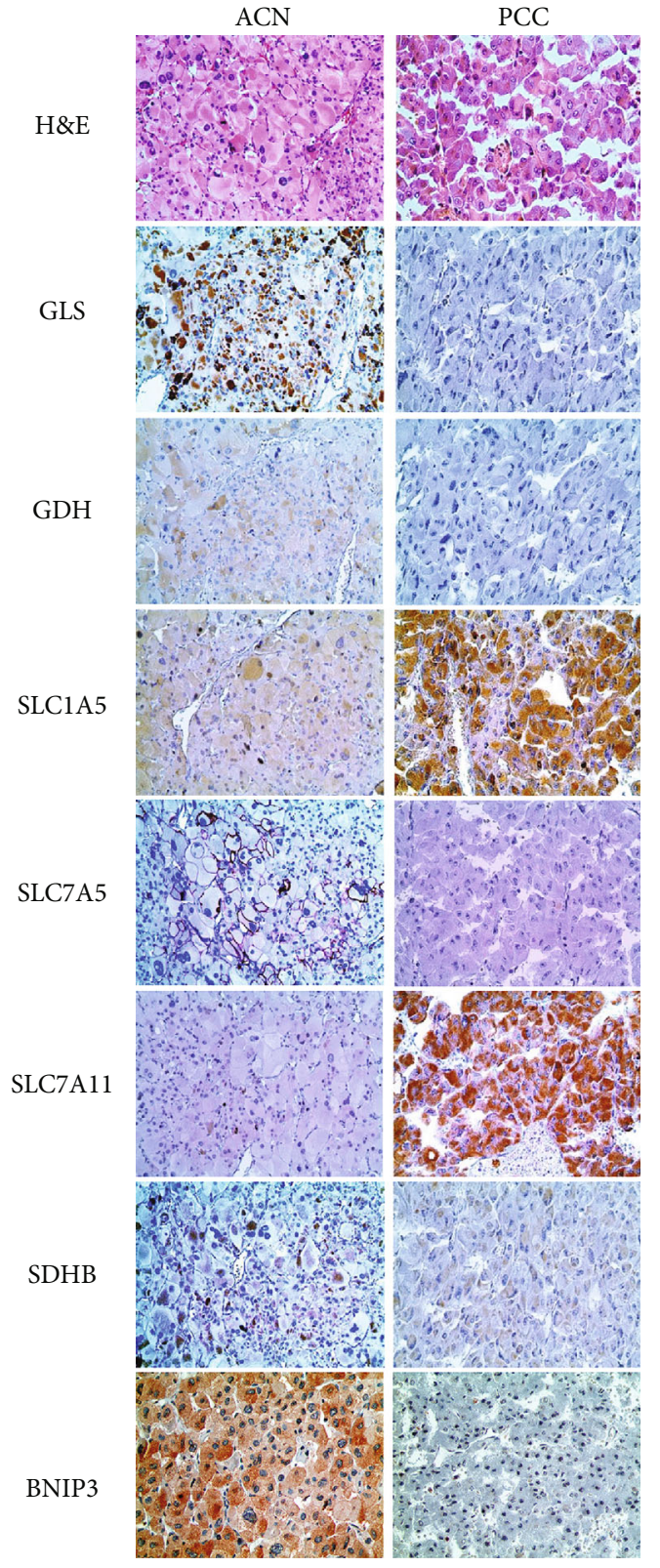

FIGURE 2: Expression of glutamine metabolism-related and amino acid transporter proteins in adrenal gland neoplasms. The expression of GLS, SLC7A5, and SDHB was higher in adrenal cortical neoplasms, whereas the expression of SLC1A5 and SLC7A11 was higher in pheochromocytomas.

to altered metabolism, we evaluated the expression of glutamine metabolism-related proteins and amino acid transporter proteins according to BNIP3 status. In ACN, BNIP3 positivity was associated with higher expression of GDH, SLC1A5, SLC7A5, and SLC7A11 $(p=0.024, p=0.002, p<$ 0.001 , and $p<0.001$ ). Meanwhile, BNIP3 positivity was associated with the increased expression of GLS and GDH in PCC $(p=0.008$ and $p=0.003)$ (Table 4$)$.

3.5. Impact of the Expression of Glutamine MetabolismRelated and Amino Acid Transporter Proteins on Patient Prognosis in Adrenal Gland Neoplasms. In Kaplan-Meier analysis, patients with ACC had shorter DFS and OS compared to ACA (all $p<0.001$ ) (Supplementary Figure 1). Additionally, GLS positivity $(p=0.039)$ and SDHB negativity $(p=0.005)$ were associated with shorter OS in ACNs. In a subgroup analysis of PCC patients with GAPP score $\geq 3$, GLS positivity $(p=0.001)$ and SDHB positivity $(p=0.001)$ were associated with shorter DFS, whereas GLS positivity $(p=0.004)$ was associated with shorter OS (Figure 4).

\section{Discussion}

In the present study, we investigated the expression of glutamine metabolism-related and amino acid transporter proteins in ACNs and PCCs in the adrenal gland. Of note, GLS, SLC7A5, and SDHB were highly expressed in ACNs, and SLC1A5 and SLC7A11 were highly expressed in PCCs. Glutamine metabolism and amino acid transport in adrenal gland neoplasms have been rarely studied; therefore, it is difficult to compare our results with those of previous studies.

Several hypotheses could apply to the differential expression of glutamine metabolism-related and amino acid transporter proteins in ACNs and PCCs. Generally, ACC is detected using ${ }^{18} \mathrm{~F}$-fludeoxyglucose (FDG) positron emission tomography-computed tomography (PET-CT) and PCC by ${ }^{18}$ F-fluorodopa $\left({ }^{18} \mathrm{~F}\right.$-DOPA $)$ PET-CT [21, 22]. ${ }^{18} \mathrm{~F}$-FDG PET-CT is used to detect glycolysis within a tumor, and glycolysis may be more active in ACNs than in PCCs because of the higher expression levels of GLS1 in ACNs than PCCs. Conversely, ${ }^{18} \mathrm{~F}$-FDOPA is a fluorinated analog of the naturally occurring amino acid L-DOPA. It is transported into a cell by the amino acid transporter system $\mathrm{L}$, which is composed of CD98hc encoded by the SLC3A2 gene and LAT1 and LAT2 encoded by the SLC7A5 and SLC7A8 genes [23]. Therefore, the expression levels of amino acid transporter proteins with similar functions, such as SLC1A5 and SLC7A11, are higher in PCCs than in ACNs.

Previous studies have shown that nearly $40 \%$ of PCCs are genetically inherited neoplasms, associated with unique metabolic abnormalities, oxygen sensing, hypermethylation, DNA repair, upregulation of specific transporters and/or receptors, and enzymes involved in the TCA cycle [24-28]. SDHx is a representative genetic variant commonly found in PCCs, and mutations in SDHx result in abnormalities in the TCA cycle that lead to altered metabolism $[23,29]$. Surprisingly, we found that SDHB positivity is associated with GLS, SLC1A5, and SLC7A1 positivity in PCCs, and 75.9\% of patients were found to have SDHB negativity in immunohistochemical staining. Given that SDHB gene mutation is present in approximately 6-9\% of PCCs [30], this is rather contradictory to the previous results that showed negative staining for SDHB when SDHB mutation is present [31, 32]. Several possible explanations could be given regarding this inconsistency. First, to minimize interobserver bias between pathologists, we established a uniform standard to define positivity of immunohistochemical staining, which could have affected the interpretation of data. Second, SDHB staining was performed by using commercial monoclonal antibodies, and the difference of the properties of antibodies 
TABLE 2: Expression of glutamine metabolism-related proteins and amino acid transporter proteins in adrenal cortical neoplasm.

\begin{tabular}{|c|c|c|c|c|}
\hline Parameters & $\begin{array}{c}\text { Total } \\
N=132(\%)\end{array}$ & $\begin{array}{c}\text { Adrenal cortical adenoma } \\
\qquad n=115(\%)\end{array}$ & $\begin{array}{l}\text { Adrenal cortical carcinoma } \\
\qquad n=17(\%)\end{array}$ & $p$ value \\
\hline GLS & & & & 0.110 \\
\hline Negative & $85(64.4)$ & $77(67.0)$ & $8(47.1)$ & \\
\hline Positive & $47(35.6)$ & $38(33.0)$ & $9(52.9)$ & \\
\hline GDH & & & & 0.114 \\
\hline Negative & $130(98.5)$ & $114(99.1)$ & $16(94.1)$ & \\
\hline Positive & $2(1.5)$ & $1(0.9)$ & $1(5.9)$ & \\
\hline SLC1A5 & & & & 0.141 \\
\hline Negative & $107(81.1)$ & $91(79.1)$ & $16(94.1)$ & \\
\hline Positive & $25(5.9)$ & $24(20.9)$ & $1(5.9)$ & \\
\hline SLC7A5 & & & & 0.124 \\
\hline Negative & $127(96.2)$ & $112(97.4)$ & $15(88.2)$ & \\
\hline Positive & $5(3.8)$ & $3(2.6)$ & $2(11.8)$ & \\
\hline SLC7A11 & & & & 0.335 \\
\hline Negative & $126(95.5)$ & $109(94.8)$ & $17(100.0)$ & \\
\hline Positive & $6(4.5)$ & $6(5.2)$ & $0(0.0)$ & \\
\hline SDHB & & & & 0.007 \\
\hline Negative & 47 (35.6) & $36(31.3)$ & $11(64.7)$ & \\
\hline Positive & $85(64.4)$ & 79 (68.7) & $6(35.3)$ & \\
\hline BNIP3 & & & & $<0.001$ \\
\hline Negative & $111(84.1)$ & $106(92.2)$ & $5(29.4)$ & \\
\hline Positive & $21(15.9)$ & $9(7.8)$ & $12(70.6)$ & \\
\hline
\end{tabular}

TABLE 3: Expression of glutamine metabolism-related proteins and amino acid transporter proteins according to SDHB status in pheochromocytoma.

\begin{tabular}{lccc}
\hline Parameters & $\begin{array}{c}\text { SDHB negative } \\
n=142(\%)\end{array}$ & $\begin{array}{c}\text { SDHB positive } \\
n=47(\%)\end{array}$ & $p$ value \\
\hline GLS & & $33(70.2)$ & $<0.001$ \\
Negative & $130(91.5)$ & $14(29.8)$ & \\
Positive & $12(8.5)$ & $45(95.7)$ & 0.153 \\
GDH & & $2(4.3)$ & \\
Negative & $141(99.3)$ & $27(57.4)$ & 0.014 \\
Positive & $1(0.7)$ & $20(42.6)$ & \\
SLC1A5 & & & \\
Negative & $108(76.1)$ & $0(100.0)$ & \\
Positive & $34(23.9)$ & $0(0.0)$ & \\
SLC7A5 & & $24(51.1)$ & \\
Negative & $142(100.0)$ & $23(48.9)$ & \\
Positive & $0(0.0)$ & $43(91.5)$ & 0.066 \\
SLC7A11 & & $4(8.5)$ & \\
Negative & $109(76.8)$ & & \\
Positive & $33(23.2)$ & & \\
BNIP3 & $139(97.9)$ & & \\
Negative & $3(2.1)$ & & \\
Positive & & & \\
\hline
\end{tabular}

could have affected the immunohistochemical staining results. Third, a difference of ethnicity and geographic difference could be present in pheochromocytoma. However, the underlying cause of this discrepancy and its association with the expression of glutamine metabolism-related and amino acid transporter proteins should be further investigated.

Fuhrman grading is a well-known but poor prognostic indicator in adrenal gland neoplasms $[17,18]$. In this study, GLS1 positivity was associated with higher Fuhrman grade and poorer prognosis in both ACNs and PCCs. Because tumor aggressiveness is associated with higher tumor metabolic activity and the expression of glutamine metabolismrelated proteins, these findings imply that evaluation of GLS1 positivity could provide useful information regarding patient prognosis. However, we found that SDHB negativity, which is known to be a negative prognostic factor in PCCs [32], was associated with shorter OS in ACNs. Although the discrepant finding that was found in our study could be also related to the fact that we only included PCC with GAPP score $\geq 3$ in our analysis for prognostic factors, it is also possible that the difference in defining SDHB positivity could have affected the results.

The glutamine metabolism pathway could be a possible target to control key enzymes and reduce glutamine uptake. GLS1 inhibitors such as CB-839 [33, 34], BPTES [35-37], and 968 [38-40] are currently in preclinical and clinical trials for the treatment of various cancers. Further investigations will be required to understand inhibition of GLS1 in adrenal gland neoplasms, including the effect of glutamine metabolism 

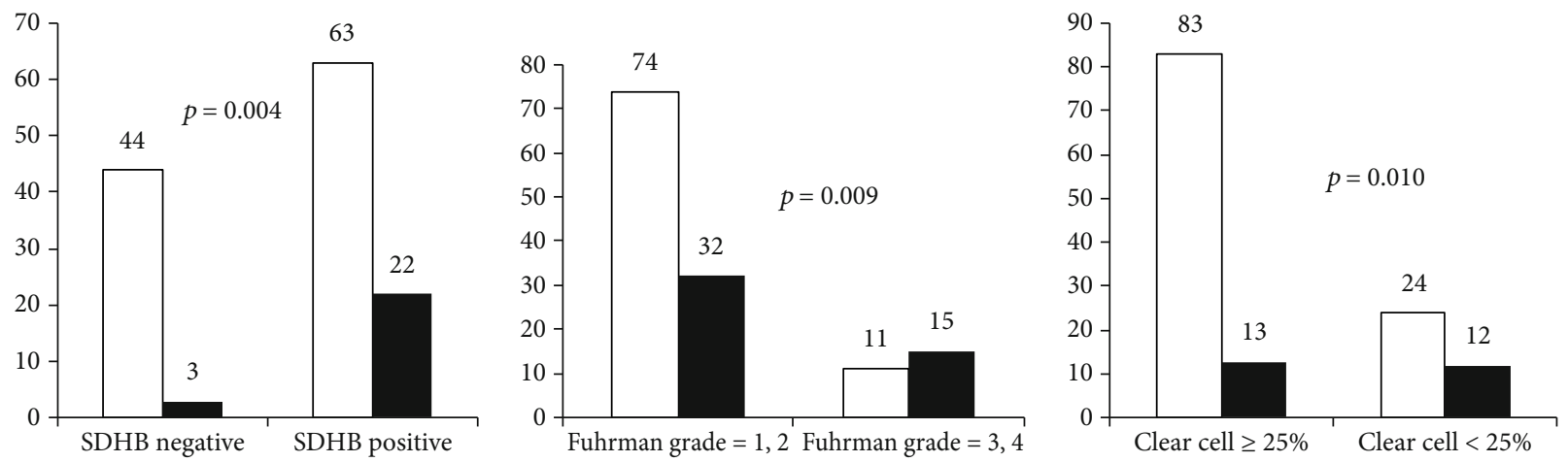

SLC1A5 negative

$\square$ GLS negative

SLC1A5 positive

- GLS positive

$\square$ SLC1A5 negative

SLC1A5 positive

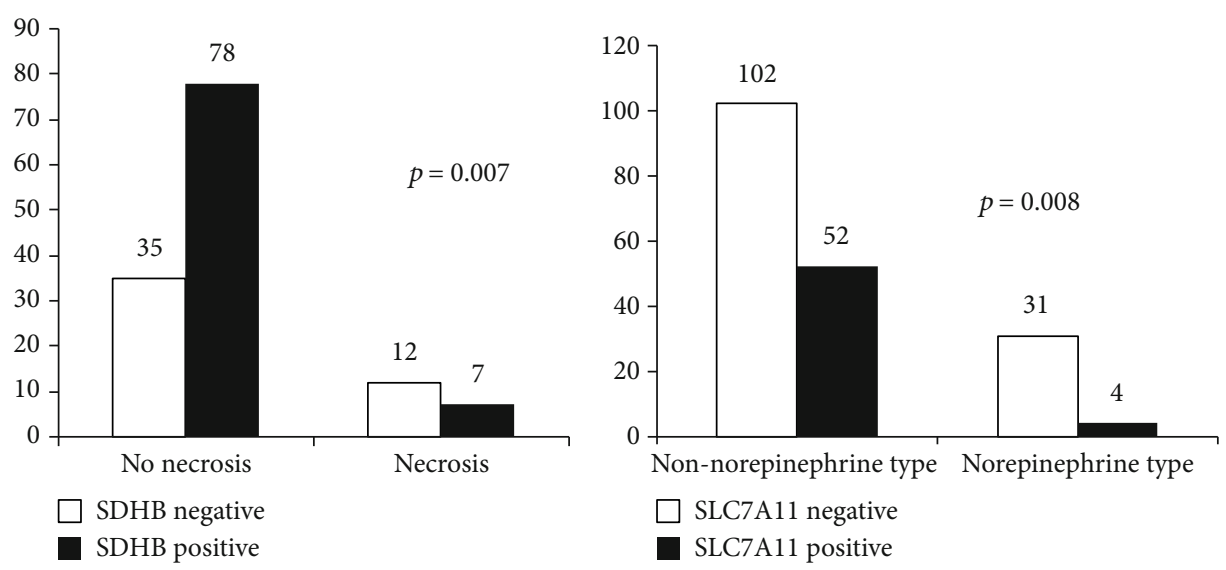

Figure 3: Correlation between the expression levels of glutamine metabolism-related and amino acid transporter proteins and clinicopathologic factors. In adrenal cortical neoplasms, GLS positivity was associated with a higher Fuhrman grade, and SLC1A5 positivity was associated with SDHB positivity and a clear cell proportion $<25 \%$. Furthermore, SDHB negativity was associated with tumor cell necrosis. In pheochromocytomas, SLC7A11 positivity was associated with nonnorepinephrine types of tumors.

TABLE 4: Expression of glutamine metabolism-related proteins and amino acid transporter proteins according to BNIP3 status in adrenal gland neoplasm.

\begin{tabular}{|c|c|c|c|c|c|c|}
\hline \multirow[b]{2}{*}{ Parameters } & \multicolumn{3}{|c|}{ Adrenal cortical neoplasm } & \multicolumn{3}{|c|}{ Pheochromocytoma } \\
\hline & $\begin{array}{c}\text { BNIP3 negative } \\
n=111(\%)\end{array}$ & $\begin{array}{c}\text { BNIP3 positive } \\
n=21(\%)\end{array}$ & $p$ value & $\begin{array}{c}\text { BNIP3 negative } \\
n=182(\%)\end{array}$ & $\begin{array}{c}\text { BNIP3 positive } \\
n=7(\%)\end{array}$ & $p$ value \\
\hline GLS & & & 0.080 & & & 0.008 \\
\hline Negative & $75(67.6)$ & $10(47.6)$ & & $160(87.9)$ & $3(42.9)$ & \\
\hline Positive & $36(32.4)$ & $11(52.4)$ & & $22(12.1)$ & $4(57.1)$ & \\
\hline GDH & & & 0.024 & & & 0.003 \\
\hline Negative & $111(100.0)$ & $19(90.5)$ & & $181(99.5)$ & $5(71.4)$ & \\
\hline Positive & $0(0.0)$ & $2(9.5)$ & & $1(0.5)$ & $2(28.6)$ & \\
\hline SLC1A5 & & & 0.002 & & & 0.410 \\
\hline Negative & $95(85.6)$ & $12(57.1)$ & & $131(72.0)$ & $4(57.1)$ & \\
\hline Positive & $16(14.4)$ & $9(42.9)$ & & $51(28.0)$ & $3(42.9)$ & \\
\hline SLC7A5 & & & $<0.001$ & & & $\mathrm{n} / \mathrm{a}$ \\
\hline Negative & $111(100.0)$ & $16(76.2)$ & & $182(100.0)$ & $7(100.0)$ & \\
\hline Positive & $0(0.0)$ & $5(23.8)$ & & $0(0.0)$ & $0(0.0)$ & \\
\hline SLC7A11 & & & $<0.001$ & & & 0.425 \\
\hline Negative & $111(100.0)$ & $15(71.4)$ & & $129(70.9)$ & $4(57.1)$ & \\
\hline Positive & $0(0.0)$ & $6(28.6)$ & & $53(29.1)$ & $3(42.9)$ & \\
\hline
\end{tabular}




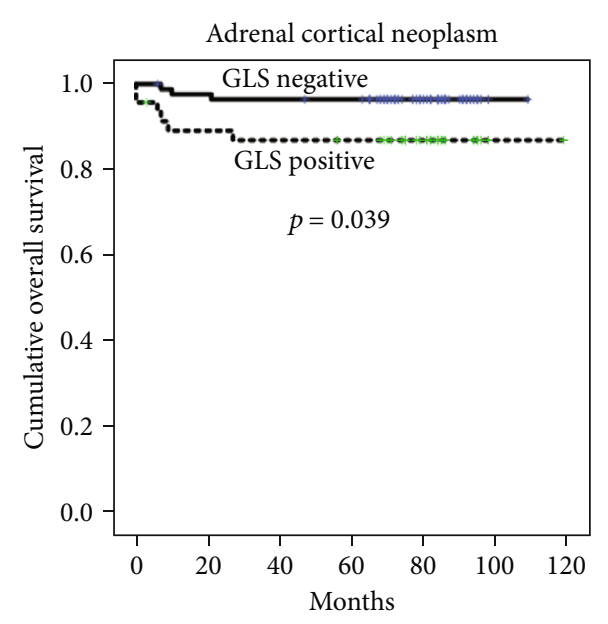

(a)

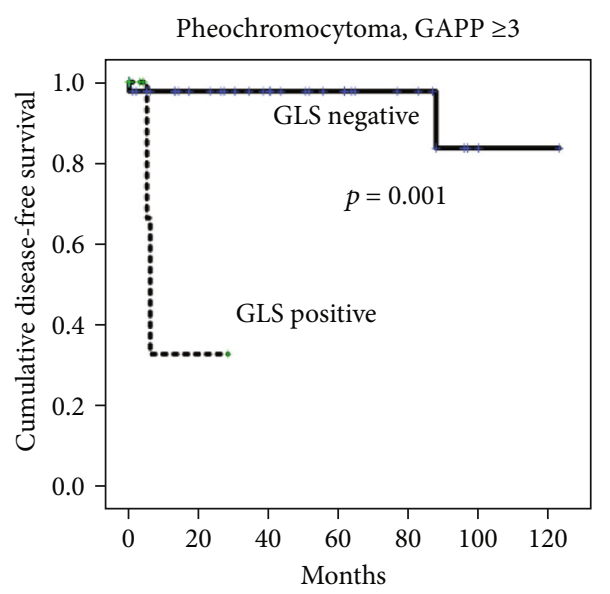

(c)

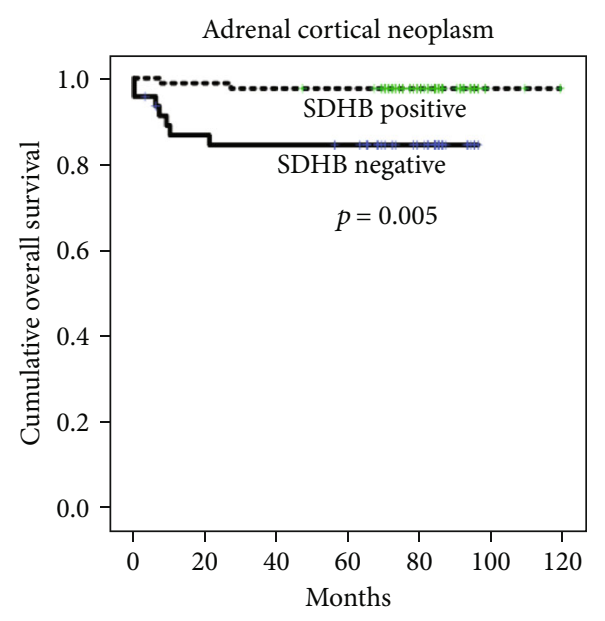

(b)

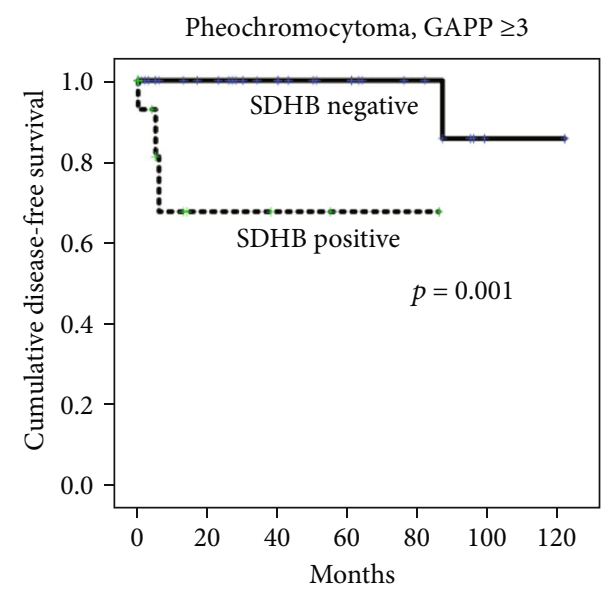

(d)

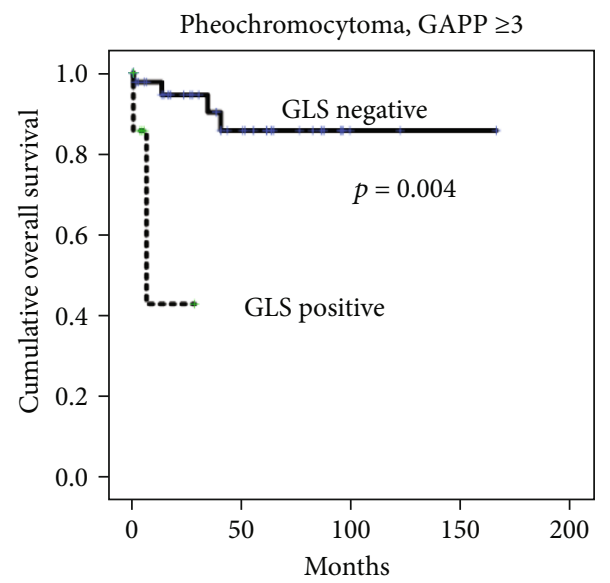

(e)

Figure 4: Impact of the expression of glutamine metabolism-related and amino acid transporter proteins on the prognosis of patients with adrenal gland neoplasms. In a univariate analysis, shorter overall survival was associated with GLS positivity $(p=0.039)$ and SDHB negativity $(p=0.005)$ in adrenal cortical neoplasms. In a subgroup analysis of PCC patients with GAPP scores $\geq 3$, GLS positivity $(p=0.001)$ and SDHB positivity $(p=0.001)$ were associated with shorter disease-free survival in univariate analysis, whereas GLS positivity $(p=0.004)$ was associated with shorter overall survival.

inhibition in ACNs, which show high glutamine metabolic activity. Alternatively, experimental evidence suggests that inhibition of amino acid transporters halts the growth of can- cer cells and promotes cancer cell death [41-43]. For example, the SLC1A5 inhibitor benzylserine (Benser) hampers the proliferation of tumor cells in human gastric cancers and in 
melanoma cell lines $[44,45]$. A selective SLC7A5 inhibitor, JPH203, has been shown to suppress cancer cell growth. In addition, JPH203 did not affect normal cells in vivo, suggesting that it can be used as a treatment agent $[46,47]$.

The advantage of this study is that it is the first to evaluate markers associated with glutamine metabolism in a large number of pathologically confirmed adrenal gland neoplasm, which is rare. However, this study also has several limitations. First, the expression of glutamine metabolism-related and amino acid transporter proteins was evaluated using TMA samples. In particular, as SDHB immunohistochemical staining, but not SDHB gene expression, was used to divide patients with PCC, this could have affected the interpretation of the results. Furthermore, the adoption of different cut-off to define SDHB immunohistochemical staining positivity and negativity compared to the previous studies [32, 48] should be also taken into account. Second, the clinical characteristics of neoplasms in the study were assessed retrospectively and genetic status could not be evaluated. Third, the number of ACC cases included in ACNs was small, and the heterogeneity of tumors included might have influenced the results. Finally, because tumor hypoxia is a factor related to altered glutamine metabolism, the changes in the tumor microenvironment could be also related to the expression of glutamine metabolism-related proteins and amino acid transporter proteins investigated.

Taken together, the expression of glutamine metabolismrelated and amino acid transporter proteins is distinct between ACNs and PCCs and is associated with prognosis. Targeting glutamine metabolism-related and amino acid transporter proteins may hold potential therapeutic value for adrenal gland neoplasms.

\section{Data Availability}

The data used to support the findings of this study are available from the corresponding author upon request.

\section{Conflicts of Interest}

The authors declare that they have no conflict of interest regarding the publication of this article.

\section{Acknowledgments}

This study was supported by a faculty research grant from Yonsei University College of Medicine for 2016 (6-20160163).

\section{Supplementary Materials}

Supplementary Figure 1: impact of the expression of glutamine metabolism-related and amino acid transporter proteins on patient prognosis in adrenal gland neoplasm. In Kaplan-Meier analysis, patients with ACC had shorter DFS and OS compared to ACA (all $p<0.001$ ). Supplementary Table 1: source, clone, and dilution of used antibodies. Supplementary Table 2: basal characteristics of adrenal cortical neoplasm. Supplementary Table 3: basal characteristics of pheochromocytoma. (Supplementary Materials)

\section{References}

[1] S. Jasim and M. A. Habra, "Management of adrenocortical carcinoma," Current Oncology Reports, vol. 21, no. 3, p. 20, 2019.

[2] A. M. Lerario, A. Moraitis, and G. D. Hammer, "Genetics and epigenetics of adrenocortical tumors," Molecular and Cellular Endocrinology, vol. 386, no. 1-2, pp. 67-84, 2014.

[3] J. W. Lenders, G. Eisenhofer, M. Mannelli, and K. Pacak, "Phaeochromocytoma," The Lancet (London, England), vol. 366, no. 9486, pp. 665-675, 2005.

[4] A. A. Cluntun, M. J. Lukey, R. A. Cerione, and J. W. Locasale, "Glutamine metabolism in cancer: understanding the heterogeneity," Trends in Cancer, vol. 3, no. 3, pp. 169-180, 2017.

[5] Y. K. Choi and K. G. Park, "Targeting glutamine metabolism for cancer treatment," Biomolecules \& Therapeutics, vol. 26, no. 1, pp. 19-28, 2018.

[6] C. M. Metallo, P. A. Gameiro, E. L. Bell et al., "Reductive glutamine metabolism by IDH1 mediates lipogenesis under hypoxia," Nature, vol. 481, no. 7381, pp. 380-384, 2012.

[7] M. Yudkoff, D. Pleasure, L. Cregar et al., "Glutathione turnover in cultured astrocytes: studies with $\left[{ }^{15} \mathrm{~N}\right]$ glutamate," Journal of Neurochemistry, vol. 55, no. 1, pp. 137-145, 1990.

[8] R. J. DeBerardinis and T. Cheng, "Q's next: the diverse functions of glutamine in metabolism, cell biology and cancer," Oncogene, vol. 29, no. 3, pp. 313-324, 2010.

[9] Y. D. Bhutia, E. Babu, S. Ramachandran, and V. Ganapathy, "Amino acid transporters in cancer and their relevance to "glutamine addiction": novel targets for the design of a new class of anticancer drugs," Cancer Research, vol. 75, no. 9, pp. 17821788, 2015.

[10] E. A. Ananieva and A. C. Wilkinson, "Branched-chain amino acid metabolism in cancer," Current Opinion in Clinical Nutrition and Metabolic Care, vol. 21, no. 1, pp. 64-70, 2018.

[11] J. Zhang, N. N. Pavlova, and C. B. Thompson, "Cancer cell metabolism: the essential role of the nonessential amino acid, glutamine," The EMBO Journal, vol. 36, no. 10, pp. 13021315, 2017.

[12] D. R. Wise and C. B. Thompson, "Glutamine addiction: a new therapeutic target in cancer," Trends in Biochemical Sciences, vol. 35, no. 8, pp. 427-433, 2010.

[13] C. V. Dang, "Rethinking the Warburg effect with Myc micromanaging glutamine metabolism," Cancer Research, vol. 70, no. 3, pp. 859-862, 2010.

[14] E. S. Schweikhard and C. M. Ziegler, "Amino acid secondary transporters: toward a common transport mechanism," Current Topics in Membranes, vol. 70, pp. 1-28, 2012.

[15] S. Broer and M. Palacin, "The role of amino acid transporters in inherited and acquired diseases," The Biochemical Journal, vol. 436, no. 2, pp. 193-211, 2011.

[16] V. Ganapathy, M. Thangaraju, and P. D. Prasad, "Nutrient transporters in cancer: relevance to Warburg hypothesis and beyond," Pharmacology \& Therapeutics, vol. 121, no. 1, pp. 29-40, 2009.

[17] L. M. Weiss, "Comparative histologic study of 43 metastasizing and nonmetastasizing adrenocortical tumors," The American Journal of Surgical Pathology, vol. 8, no. 3, pp. 163-170, 1984.

[18] L. M. Weiss, L. J. Medeiros, and A. L. Vickery Jr., "Pathologic features of prognostic significance in adrenocortical carcinoma," The American Journal of Surgical Pathology, vol. 13, no. 3, pp. 202-206, 1989. 
[19] N. Kimura, R. Takayanagi, N. Takizawa et al., "Pathological grading for predicting metastasis in phaeochromocytoma and paraganglioma," Endocrine-Related Cancer, vol. 21, no. 3, pp. 405-414, 2014.

[20] L. R. Henry, H. O. Lee, J. S. Lee et al., "Clinical implications of fibroblast activation protein in patients with colon cancer," Clinical Cancer Research: An Official Journal of the American Association for Cancer Research, vol. 13, no. 6, pp. 1736-1741, 2007.

[21] D. Taïeb, L. Tessonnier, F. Sebag et al., "The role of ${ }^{18} \mathrm{~F}$ FDOPA and ${ }^{18} \mathrm{~F}$-FDG-PET in the management of malignant and multifocal phaeochromocytomas," Clinical Endocrinology, vol. 69, no. 4, pp. 580-586, 2008.

[22] S. Barollo, L. Bertazza, S. Watutantrige-Fernando et al., "Overexpression of L-type amino acid transporter 1 (LAT1) and 2 (LAT2): novel markers of neuroendocrine tumors," PLoS One, vol. 11, no. 5, article e0156044, 2016.

[23] C. C. Feral, F. S. Tissot, L. Tosello et al., " ${ }^{18}$ F-fluorodihydroxyphenylalanine PET/CT in pheochromocytoma and paraganglioma: relation to genotype and amino acid transport system L," European Journal of Nuclear Medicine and Molecular Imaging, vol. 44, no. 5, pp. 812-821, 2017.

[24] I. Jochmanova and K. Pacak, "Pheochromocytoma: the first metabolic endocrine cancer," Clinical Cancer Research: An Official Journal of the American Association for Cancer Research, vol. 22, no. 20, pp. 5001-5011, 2016.

[25] E. Letouzé, C. Martinelli, C. Loriot et al., "SDH mutations establish a hypermethylator phenotype in paraganglioma," Cancer Cell, vol. 23, no. 6, pp. 739-752, 2013.

[26] I. Jochmanova, C. Yang, Z. Zhuang, and K. Pacak, "Hypoxiainducible factor signaling in pheochromocytoma: turning the rudder in the right direction," Journal of the National Cancer Institute, vol. 105, no. 17, pp. 1270-1283, 2013.

[27] Y. C. Chae, A. Angelin, S. Lisanti et al., "Landscape of the mitochondrial Hsp90 metabolome in tumours," Nature Communications, vol. 4, no. 1, article 2139, 2013.

[28] A. A. de Cubas, E. Korpershoek, L. Inglada-Pérez et al., "DNA methylation profiling in pheochromocytoma and paraganglioma reveals diagnostic and prognostic markers," Clinical Cancer Research: An Official Journal of the American Association for Cancer Research, vol. 21, no. 13, pp. 3020-3030, 2015.

[29] A. Jha, K. de Luna, C. A. Balili et al., "Clinical, diagnostic, and treatment characteristics of SDHA-related metastatic pheochromocytoma and paraganglioma," Frontiers in Oncology, vol. 9, p. 53, 2019.

[30] B. Pasini and C. A. Stratakis, "SDH mutations in tumorigenesis and inherited endocrine tumours: lesson from the phaeochromocytoma-paraganglioma syndromes," Journal of Internal Medicine, vol. 266, no. 1, pp. 19-42, 2009.

[31] N. Kimura, K. Takekoshi, and M. Naruse, "Risk stratification on pheochromocytoma and paraganglioma from laboratory and clinical medicine," Journal of Clinical Medicine, vol. 7, no. 9, p. 242, 2018.

[32] S. Hescot, M. Curras-Freixes, T. Deutschbein et al., "Prognosis of malignant pheochromocytoma and paraganglioma (MAPPProno study): a European network for the study of adrenal tumors retrospective study," The Journal of Clinical Endocrinology and Metabolism, vol. 104, no. 6, pp. 2367-2374, 2019.

[33] M. I. Gross, S. D. Demo, J. B. Dennison et al., "Antitumor activity of the glutaminase inhibitor CB-839 in triplenegative breast cancer," Molecular Cancer Therapeutics, vol. 13, no. 4, pp. 890-901, 2014.
[34] N. Jacque, A. M. Ronchetti, C. Larrue et al., "Targeting glutaminolysis has antileukemic activity in acute myeloid leukemia and synergizes with BCL-2 inhibition," Blood, vol. 126, no. 11, pp. 1346-1356, 2015.

[35] E. W. Hartwick and N. P. Curthoys, "BPTES inhibition of hGA (124-551), a truncated form of human kidney-type glutaminase," Journal of Enzyme Inhibition and Medicinal Chemistry, vol. 27, no. 6, pp. 861-867, 2012.

[36] Y. Xiang, Z. E. Stine, J. Xia et al., "Targeted inhibition of tumor-specific glutaminase diminishes cell-autonomous tumorigenesis," The Journal of Clinical Investigation, vol. 125, no. 6, pp. 2293-2306, 2015.

[37] A. Emadi, S. A. Jun, T. Tsukamoto, A. T. Fathi, M. D. Minden, and C. V. Dang, "Inhibition of glutaminase selectively suppresses the growth of primary acute myeloid leukemia cells with IDH_ mutations," Experimental Hematology, vol. 42, no. 4, pp. 247-251, 2014.

[38] J. W. Erickson and R. A. Cerione, "Glutaminase: a hot spot for regulation of cancer cell metabolism?," Oncotarget, vol. 1, no. 8, pp. 734-740, 2010.

[39] W. P. Katt, M. A. Antonyak, and R. A. Cerione, "Simultaneously targeting tissue transglutaminase and kidney type glutaminase sensitizes cancer cells to acid toxicity and offers new opportunities for therapeutic intervention," Molecular Pharmaceutics, vol. 12, no. 1, pp. 46-55, 2014.

[40] N. E. Simpson, V. P. Tryndyak, M. Pogribna, F. A. Beland, and I. P. Pogribny, "Modifying metabolically sensitive histone marks by inhibiting glutamine metabolism affects gene expression and alters cancer cell phenotype," Epigenetics, vol. 7, no. 12, pp. 1413-1420, 2014.

[41] A. N. McCracken and A. L. Edinger, "Nutrient transporters: the Achilles' heel of anabolism," Trends in Endocrinology and Metabolism: TEM, vol. 24, no. 4, pp. 200-208, 2013.

[42] Y. Cormerais, P. A. Massard, M. Vucetic et al., "The glutamine transporter ASCT2 (SLC1A5) promotes tumor growth independently of the amino acid transporter LAT1 (SLC7A5)," The Journal of Biological Chemistry, vol. 293, no. 8, pp. 2877-2887, 2018.

[43] Q. Wang and J. Holst, "L-type amino acid transport and cancer: targeting the mTORC1 pathway to inhibit neoplasia," American Journal of Cancer Research, vol. 5, no. 4, pp. 12811294, 2015.

[44] J. Ye, Q. Huang, J. Xu et al., "Targeting of glutamine transporter ASCT2 and glutamine synthetase suppresses gastric cancer cell growth," Journal of Cancer Research and Clinical Oncology, vol. 144, no. 5, pp. 821-833, 2018.

[45] Q. Wang, K. A. Beaumont, N. J. Otte et al., "Targeting glutamine transport to suppress melanoma cell growth," International Journal of Cancer, vol. 135, no. 5, pp. 1060-1071, 2014.

[46] K. Oda, N. Hosoda, H. Endo et al., "L-type amino acid transporter 1 inhibitors inhibit tumor cell growth," Cancer Science, vol. 101, no. 1, pp. 173-179, 2010.

[47] C. Rosilio, M. Nebout, V. Imbert et al., "L-type amino-acid transporter 1 (LAT1): a therapeutic target supporting growth and survival of T-cell lymphoblastic lymphoma/T-cell acute lymphoblastic leukemia," Leukemia, vol. 29, no. 6, pp. 1253 1266, 2015.

[48] A. J. Gill, D. E. Benn, A. Chou et al., "Immunohistochemistry for SDHB triages genetic testing of $S D H B, S D H C$, and $S D H D$ in paraganglioma-pheochromocytoma syndromes," Human Pathology, vol. 41, no. 6, pp. 805-814, 2010. 